\title{
Sexually transmitted infections manifesting as proctitis
}

\author{
Chris A Lamb, ${ }^{1,2}$ Elizabeth Iris Mary Lamb, ${ }^{1,3}$ John C Mansfield, ${ }^{4}$ \\ K Nathan Sankar ${ }^{5}$
}

\begin{abstract}
${ }^{1}$ Institute of Cellular Medicine, Newcastle University, Newcastle upon Tyne, UK

${ }^{2}$ Department of Gastroenterology, Newcastle upon Tyne Hospitals NHS Foundation Trust, Newcastle upon Tyne, UK

${ }^{3}$ Northern Deanery General Practice Vocational Training Scheme, Northern Deanery, North East Strategic Health Authority, Newcastle upon Tyne, UK

${ }^{4}$ Institute of Genetic Medicine, Newcastle University, Newcastle upon Tyne, UK

${ }^{5}$ Department of Genitourinary Medicine, Newcastle Primary Care Trust, Newcroft Centre, Newcastle upon Tyne Hospitals Trust, Newcastle upon Tyne, UK
\end{abstract}

\section{Correspondence to}

Dr Christopher Andrew Lamb, Institute of Cellular Medicine, The Medical School, Newcastle University, William Leech Building, Framlington Place, Newcastle upon Tyne NE2 4HH, UK; c.a.lamb@ncl.ac.uk

Received 26 October 2012 Revised 26 October 2012 Accepted 29 October 2012

To cite: Lamb CA, Lamb EIM, Mansfield JC, et al. Frontline Gastroenterology 2013, 4, 32-40.

\begin{abstract}
There is a rising incidence of several sexually transmitted infections (STIS), many of which can present with proctitis. Causative organisms include Neisseria gonorrhoeae, Chlamydia trachomatis, herpes simplex virus, Treponema pallidum (syphilis), Giardia lamblia (giardiasis) and Entamoeba histolytica (amoebiasis). This paper outlines important clinical discriminators and key investigations to distinguish these organisms from non-infective pathology that include inflammatory bowel disease, solitary rectal ulcer syndrome and Behçet's syndrome. Management of these infections is described and suggestions are made for successful gastroenterology clinical consultation when an STI is suspected.
\end{abstract}

\section{INTRODUCTION}

Patients presenting to secondary care with symptoms of diarrhoea and rectal bleeding may be found to have features consistent with inflammatory bowel disease (IBD) on endoscopic or radiological investigation. The main differential diagnoses of infectious colitis, ischaemic colitis and non-steroidal antiinflammatory drug enteropathy are well understood and widely covered in the literature. However, there are several less commonly encountered causes of intestinal inflammation that may not respond to, or be made worse by, immunosuppressant therapy intended for Crohn's disease or ulcerative colitis. Competency 2.c. of the 2010 Gastroenterology curriculum (box 1) stipulates that UK trainees must have an appreciation of the range of potential aetiologies and the investigative process of differential causes of intestinal inflammation, in order to guide appropriate and successful therapeutic intervention. In the UK and many other western countries there has been a sustained annual increase in the incidence of sexually transmitted infections (STIs) over the past decade. ${ }^{1}$ Several STIs including gonorrhoea, chlamydia, syphilis and herpes virus can manifest with symptoms and endoscopic features of proctitis. This article outlines these diagnoses and suggests key clinical features or important diagnostic tests that may aid earlier diagnosis and appropriate treatment.

\section{GONORRHOEA}

Caused by Neisseria gonorrhoeae, gonorrhoea is transmitted by direct contact of mucosal surfaces, and so proctitis is commonest in men or women participating in receptive anal intercourse, although in women it can occur due to transmucosal spread of infected genital fluid. Symptoms of proctitis are non-discriminatory including lower abdominal pain, diarrhoea, rectal bleeding and tenesmus; however, it may be associated with an anorectal purulent discharge. Diagnostic suspicion should be raised in the presence of urethral discharge and/or pharyngeal infection. $^{2}$

Anogenital samples should be sent for immediate Gram stain, in which the presence of Gram-negative diplococci are suggestive of a diagnosis with a sensitivity of $90-95 \%{ }^{2}$ (although the specificity of this test may be lower than 60\%). ${ }^{3}$ Nucleic acid amplification tests (NAAT, eg, PCR), although not yet licensed for this anatomical site ${ }^{2}$ are more sensitive than culture and based on available evidence should be used for diagnosis. In addition, anogenital and pharyngeal swabs in the appropriate transport medium (as advised by the local microbiologist) should also be sent for culture in order to determine antibiotic sensitivities. A high proportion of patients with confirmed gonococcal 


\section{Box 1 Gastroenterology curriculum 2010}

Competency 2.c. Intestinal disorders: Inflammatory and infective conditions

- Recognises the range of important inflammatory conditions of the intestine other than inflammatory bowel disease

- Knows the range of potential aetiologies including infection

- Knows the means of investigation of infectious diseases

infection will also have chlamydia, and so involvement of genitourinary medicine (GUM) specialists and testing for co-infection must be undertaken in confirmed cases.

Treatment of rectal gonorrhoea without available antibiotic sensitivities should be with ceftriaxone $500 \mathrm{mg}$ intramuscularly once, alongside azithromycin $1 \mathrm{~g}$ orally once. ${ }^{2}$ Gonorrhoea is becoming increasingly resistant to cefalosporins and so repeat testing is advisable following treatment to ensure eradication and to prevent further resistance development. Azithromycin co-treatment with ceftriaxone may improve eradication of gonorrhoea and aims to slow the development of further cephalosporin resistance.

\section{LYMPHOGRANULOMA VENEREUM}

There are several serovars of Chlamydia trachomatis. Serovars D-K may lead to superficial rectal mucosal infection (see below); however, serovars L1, L2 and L3 may lead to deeper invasive infection that can affect submucosal tissue and lymphatics, thereby allowing spread to lymph nodes. These invasive serovars are responsible for lymphogranuloma venereum (LGV), also known as lymphogranuloma inguinale, lymphopatia venereum and Durand-Nicolas-Favre's disease. ${ }^{4}$ Previously uncommon in western populations, there have been several outbreaks of this condition in Europe and North America within the past decade. Although LGV affects men and women, the majority of cases occurs in men who have sex with men (MSM). The commonest presentation of the condition is proctitis, usually within several weeks of sexual contact, with symptoms including proctalgia, rectal bleeding and mucopurulent anal discharge. Endoscopic features may mimic IBD proctitis with the additional finding of purulent exudate. Advanced infection may lead to perianal abscess, fistula formation and anorectal stricturing. LGV may also exhibit extra-intestinal manifestations including reactive arthritis and hepatitis.

Discriminating factors from IBD include urethral or vaginal mucopurulent discharge, and painful inguinal and femoral lymphadenopathy may be present that can progress to abscess formation. Low-grade pyrexia and rigors may also be discriminating signs.

GUM or infectious disease specialists should be consulted in suspected or confirmed cases of LGV. A diagnosis requires mucosal sampling using anogenital swabs and the detection of L1, L2 or L3 biovar C trachomatis by NAAT and genotyping. Testing for other STIs including HIV (which is particularly associated with LGV), hepatitis B and C, syphilis and gonorrhoea should also be undertaken in confirmed cases. Current European guidance on the management of LGV recommends doxycyline $100 \mathrm{mg}$ twice a day for 21 days first line, or erythromycin $500 \mathrm{mg}$ four times a day for 21 days. ${ }^{4}$ Symptoms should abate within 7-14 days. Patients should be advised to abstain from sexual contact until completion of therapy, and sexual partners within the preceding 3 months should be notified and screened for infection. A test of cure should be performed at least 4 weeks following completion of therapy.

\section{CHLAMYDIA}

Caused by $\mathrm{C}$ trachomatis serovars $\mathrm{D}-\mathrm{K}$, transmission of chlamydia is similar to that of $N$ gonorrhoeae. Routine screening using NAAT in GUM clinics detects rectal chlamydia in approximately $10 \%$ of MSM and $5 \%$ of women. Over $50 \%$ of chlamydial infection in MSM is rectal without urethral infection.

Approximately $70 \%$ of rectal chlamydial infections are asymptomatic; however, mild symptoms of diarrhoea and anal discharge may occur. Occasionally these symptoms can be as severe as LGV or gonorrhoea. Microscopy of anal discharge may show the presence of neutrophils but unlike gonorrhoea, chlamydia cannot be diagnosed by immediate Gram stain. Endoscopy may show a 'cobblestone' appearance due to the infiltration of underlying lymphoid follicles.

Although not licensed for extragenital sites, NAAT has been shown to be sensitive and specific and should be requested to diagnose rectal chlamydial infection. Aptima combo 2 (Hologic Gen-Probe) is a transcriptionmediated assay that has the advantage of detecting both $N$ gonorrhoeae and $C$ trachomatis. When requesting NAAT for $C$ trachomatis in the presence of proctitis, genotyping for LGV should be requested as this is not usually carried out on routine specimens.

Rectal chlamydia is best treated with doxycycline $100 \mathrm{mg}$ twice a day for 1 week. (In cases of proctitis in which LGV is a possibility a 3 -week course of doxycycline can be started pending the test results. If LGV is excluded, the treatment can be stopped after 1 week.) An alternative is azithromycin $1 \mathrm{~g}$ as a single dose but up to $20 \%$ treatment failure has been observed and therefore a test of cure with repeat NAAT after 6 weeks is recommended.

\section{HERPES SIMPLEX VIRUS}

Caused by either herpes simplex virus (HSV) type 1 or type 2, primary herpes simplex infection may be asymptomatic, but $40 \%$ of men and $70 \%$ of women will develop systemic features that include malaise, headaches and muscle pains. HSV ascends peripheral sensory nerves then lies dormant in sensory or autonomic ganglia where it can later reactivate. Recurrent 
HSV occurs in approximately $60 \%$ of those infected with type $1 \mathrm{HSV}$ and $90 \%$ in type $2 \mathrm{HSV}$. Proctitis is usually associated with anal intercourse and diagnostic suspicion should be raised in the presence of perianal vesicles or painful oral/anogenital ulceration.

PCR on rectal biopsy, or exudate from a lesion is the current ideal diagnostic modality for rectal $\mathrm{HSV}^{3}$ Recommended treatment for acute infection is 5 days of either aciclovir $200 \mathrm{mg}$ five times a day, aciclovir $400 \mathrm{mg}$ three times a day, valaciclovir $500 \mathrm{mg}$ twice a day, or famciclovir $125 \mathrm{mg}$ twice a day. ${ }^{5}$ For recurrent HSV, aciclovir may be given four times a day for up to 1 year; however, only $20 \%$ of patients will see a subsequent reduction in disease recurrence.
It should be appreciated that anogenital ulcers associated with both HSV and syphilis lead to a 1.5 to sevenfold increase in HIV transmission due to the associated mucosal barrier breach, and so HIV testing should be undertaken.

\section{SYPHILIS}

Caused by the spirochete Treponema pallidum, the incidence of syphilis both in men and women has steadily risen in recent years, especially so in MSM. ${ }^{6}$ Primary syphilis presents within 3 months of exposure (on average within 2-3 weeks), with a painless solitary ulcer (chancre, see figure 1) and regional lymphadenopathy that may affect the genitalia, mouth or rectum.

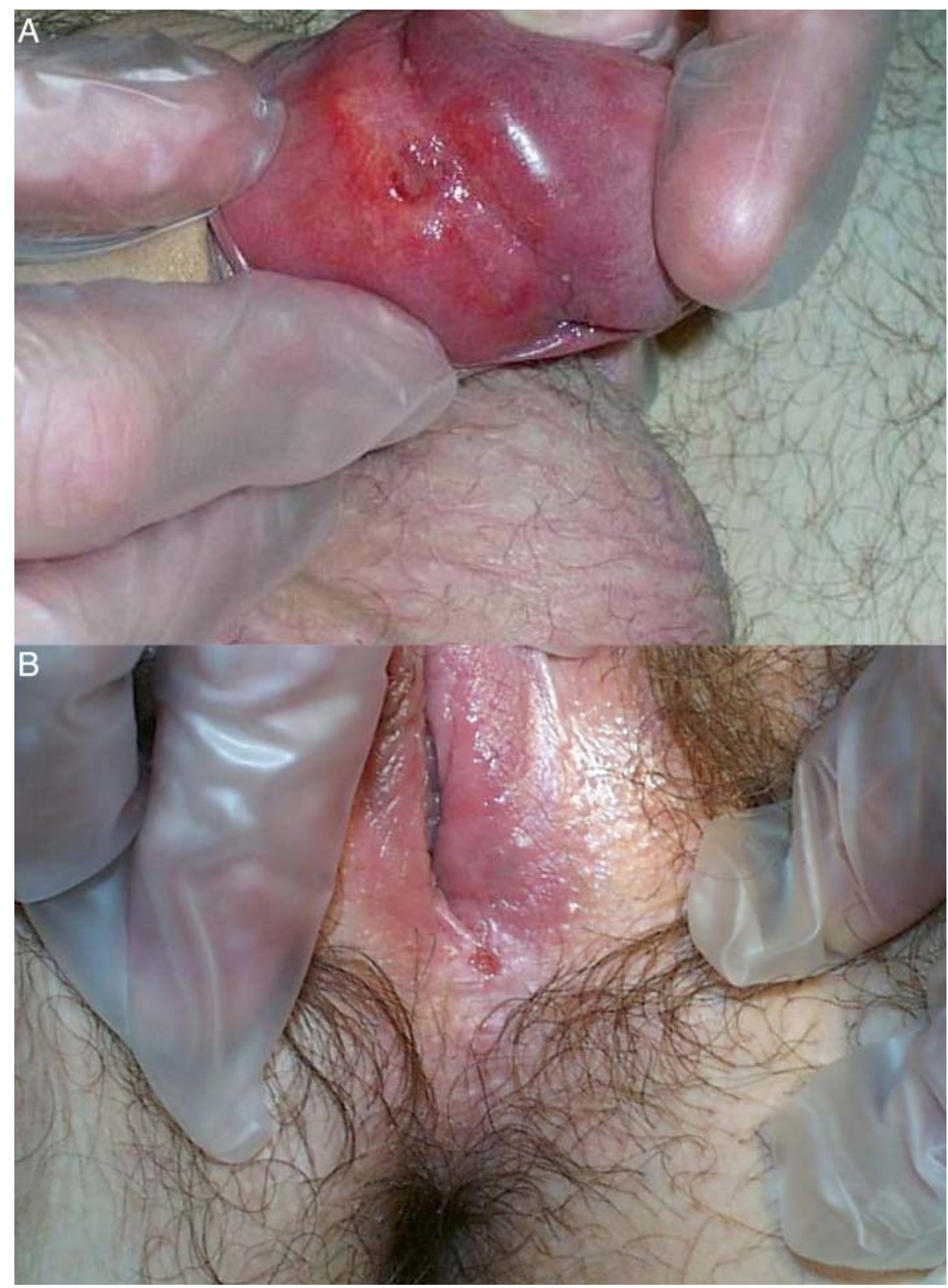

Figure 1 Solitary syphilitic chancre affecting (a) male and (b) female external genitalia. A chancre is the archetypal feature of primary syphilis. These ulcers are classically anogenital, solitary, indurated and painless; however, in atypical cases they may be multiple and painful, and can occur at extra-anogenital sites. 
These ulcers are typically indurated with a clean base and discharge serous fluid. If unidentified, primary disease can progress to secondary syphilis typically in 6-12 weeks (but up to 2 years) later, in which bacteraemia leads to multisystem disease that may include peripheral neuropathy, maculopapular rash (typically palmoplantar, see figure 2), hepatitis, glomerulonephritis, meningitis or anterior uveitis. Secondary syphilis can also manifest with oral ulceration (typically with a snail tract distribution), proctitis and condylomata lata, which are wart-like papules commonly perianal or genital in anatomical origin. Covered in a greyish exudate, condylomata lata are highly infectious. Tertiary syphilis, which may occur several years following primary or secondary disease, can have major neurological or cardiovascular sequelae. These include dementia, paresis, seizures, aortitis or aneurysms. Tertiary syphilis may also feature gumma, which are granulomatous nodules or ulcers of the gastrointestinal tract or buttocks.

Early infection up to 2 years after acquisition without any manifestations of primary or secondary stages is described as early latent syphilis. Primary, secondary and early latent stages of syphilis are infectious, whereas the later stages of tertiary syphilis are largely non-infectious. Emphasis should therefore be placed on diagnosing and treating primary, secondary and early latent syphilis promptly so as to avoid later complications.

A diagnosis of syphilis can be made by dark ground microscopy or PCR of ulcer exudate, condylomata

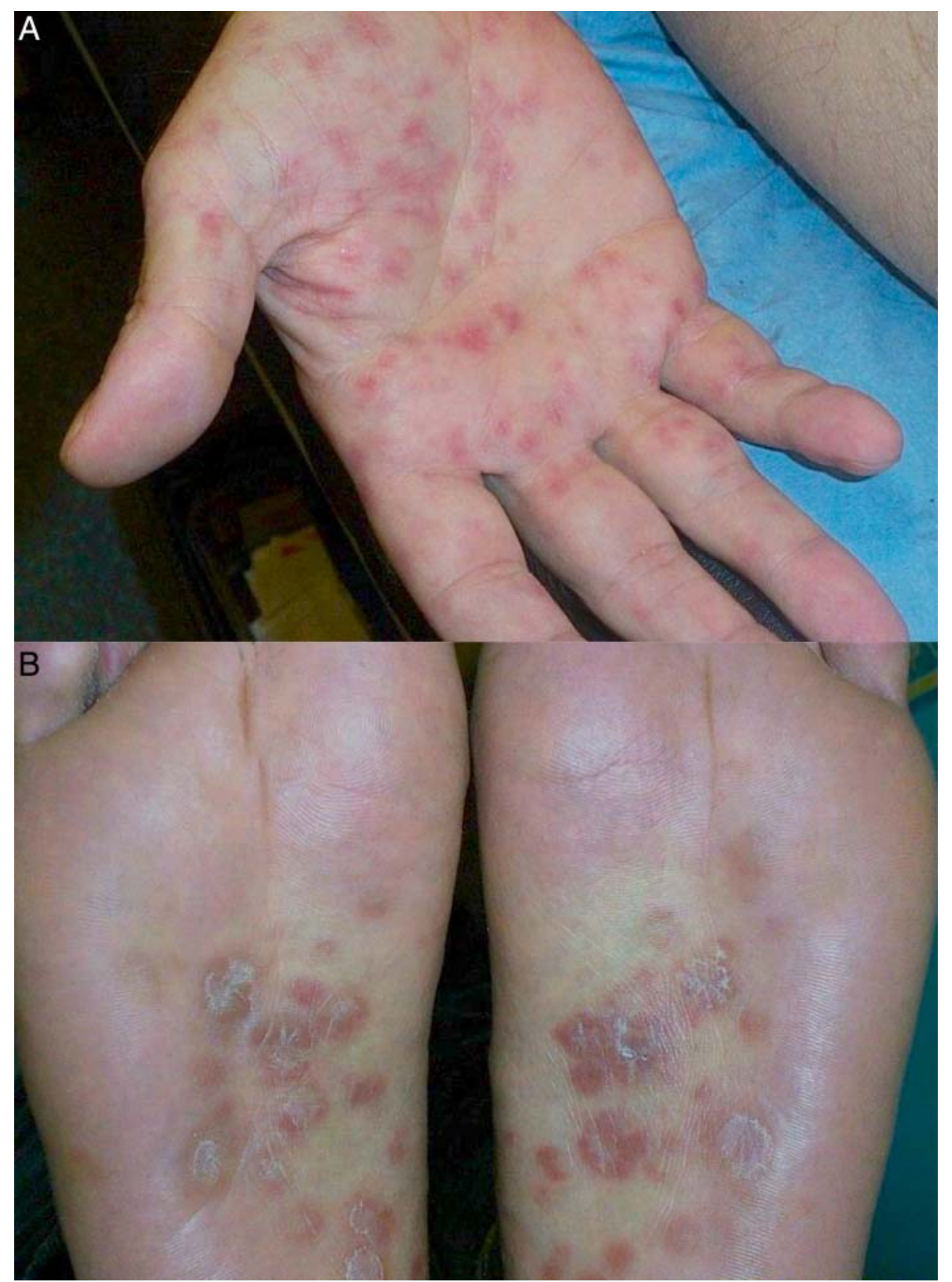

Figure 2 Palmoplantar rash in secondary syphilis. The skin eruption associated with secondary syphilis characteristically has a palmoplantar distribution, is pink or dark red and not pruritic. 
lata exudate or rectal biopsies. It may be 12 weeks from infection until antibodies become detectable within serum, and so a repeat blood sample 3 months following last sexual risk may be required. When a diagnosis is suspected or confirmed, GUM or infectious disease specialists should be consulted. Contact tracing and screening is necessary, as between $40-60 \%$ of contactable sexual partners may be infected. ${ }^{6}$

First-line treatment of primary and secondary syphilis is ideally with intramuscular benzyl benzathine penicillin 2.4 million units once. This form of penicillin is slowly hydrolysed to benzylpenicillin giving sustained release, and is advantageous as it ensures bioavailability and can be supervised. Alternatively, intramuscular procaine penicillin 600000 units a day for 10-14 days can be given. Second-line therapy is with doxycycline $100 \mathrm{mg}$ twice a day for 14 days. For tertiary syphilis prolonged courses of the above therapies may be required.

\section{OTHER SEXUALLY TRANSMITTED PATHOGENS THAT MAY CAUSE PROCTITIS}

Giardia lamblia (giardiasis) and Entamoeba histolytica (amoebiasis) may also be inoculated by sexual contact. $^{3}{ }^{7}$ Spread by the faecal oral route, sexual transmission is most commonly seen in MSM.

\section{Giardiasis}

In giardiasis, protozoa adhere and replicate on the brush border of the duodenum and jejunum, feeding on the mucous secretions of the intestine. In some cases proctitis is evident. Classically patients will experience foul-smelling steatorrhoea around 2-3 weeks following infection. Diagnosis is by direct microscopy of faeces that may reveal trophozoites (the motile active feeding stage of the protozoan life cycle) in liquid faeces. Oocysts may be seen in either liquid or solid faeces; however, they may be excreted in a cyclical manner, and so repeated stool tests or in some cases duodenal biopsy or aspirate may be required to confirm the diagnosis. Faecal antigen detection kits may also aid diagnosis. Treatment of giardiasis is with metronidazole $400 \mathrm{mg}$ three times a day for 5 days.

\section{Amoebiasis}

Diarrhoea caused by amoebiasis ensues 2-4 weeks following the ingestion of cysts, when trophozoites breach the mucosa causing an inflammatory infiltrate and subsequent proctocolitis. Diarrhoea, which can be bloody, may persist for several weeks. Diagnosis of E histolytica trophozoites or cysts is usually possible on microscopic examination of stool specimens. Treatment is with metronidazole $800 \mathrm{mg}$ three times a day or tindazole $2 \mathrm{~g}$ once a day for 5-8 days, coupled with diloxanide furoate $500 \mathrm{mg}$ three times a day for 10 days to optimise eradication. Stool samples should be analysed at monthly intervals for 3 months following therapy as a test of cure, and further treatment should be provided if persistent amoebiasis is identified.

\section{IMPORTANT NON-INFECTIOUS DIFFERENTIAL DIAGNOSES OF STI-INDUCED PROCTITIS}

While certain genital, oral or systemic features may suggest an STI cause of proctitis, it is important to be aware that there are other less commonly encountered non-infectious causes of proctitis that may display similar characteristics. These include Behçet's syndrome and solitary rectal ulcer syndrome (SRUS).

\section{Behçet's syndrome}

Classically presenting with orogenital ulceration due to an underlying vasculitis, Behçet's syndrome can also affect any part of the gastrointestinal tract. ${ }^{8}$ It may present with perianal ulceration, fistulation, segmental colitis and abdominal pain. Extra-intestinal manifestations including arthritis, anterior uveitis and erythema nodosum may lead to a misdiagnosis of Crohn's disease. This is further compounded by the possibility of granulomata being found on biopsy. The prevalence of Behçets syndrome is greatest in the middle east, far east and the Mediterranean basin and is highest in Turkey at $8-42$ per 1000 people. Within Behçet's populations the prevalence of gastrointestinal involvement varies, being highest at approximately $30 \%$ in Japan. Thrombophlebitis of the legs is frequently observed and may be a subtle differentiating feature between IBD and Behçet's syndrome. Endoscopically ulcers may appear well demarcated and 'punched out', sometimes without surrounding colitis. Histological examination of intestinal biopsies may reveal a lymphocytic infiltration, classically with vasculitis of small vessels; however, histology may be indistinguishable from IBD. A diagnosis of Behçet's syndrome is consequently made clinically and is dependent on the presence of recurrent oral ulceration plus two of recurrent genital ulceration, eye lesions (uveitis or retinal vasculitis), skin lesions (erythema nodosum, pseudofolliculitis, papulopustular lesions, or acneform nodules in post-adolescent patients), or a positive pathergy test at $24-48 \mathrm{~h}$ (a sterile skin prick test that may lead to pustule or nodule formation; however, this test is often negative). ${ }^{9}$ Treatment options for intestinal manifestations of Behçet's syndrome include 5-aminosalicylic acid, sulfasalazine, or azathioprine. Corticosteroids may be beneficial in some patients.

\section{Solitary rectal ulcer syndrome}

Commonly, SRUS presents with rectal bleeding, the passage of mucous and regularly a sensation of incomplete evacuation. ${ }^{10}$ The condition is often associated with rectal prolapse. Despite nomenclature, SRUS may present with erythema of the rectal mucosa, polypoid inflammation or multiple rather than single ulcers. Consequently, a misdiagnosis of IBD or malignancy may be made. Biopsy is therefore essential to exclude rectal adenocarcinoma, and histological features of thickened mucosa and smooth muscle 
extension from the muscularis into the lamina propria are characteristic of SRUS. In comparison to IBD, an inflammatory infiltrate is not a prominent finding on histology. Diagnosis is often possible on the basis of history and endoscopic examination; however, defecating proctography and ultrasonography can be useful aids to diagnosis. Common findings on defecating proctography include internal or external rectal prolapse, often with incomplete rectal evacuation. Endoanal ultrasound classically reveals a thickened internal anal sphincter, and may show contraction or failure of relaxation of the puborectalis muscle on straining. Treatment options have variable results and must be considered on an individual basis. These include biofeedback therapy to modify defecation behaviour, and surgical intervention including abdominal rectopexy.

\section{FURTHER CONSIDERATIONS WHEN MANAGING PROCTITIS CAUSED BY A SEXUALLY TRANSMITTED PATHOGEN}

Patients diagnosed in the gastroenterology clinic with an STI should be seen by an expert in sexual heath and associated infections, when clear information regarding their diagnosis should be provided to patients and their sexual contacts. Partner notification and screening should be undertaken (partners in preceding 3 months for primary syphilis, chlamydia, LGV, or rectal gonorrhoea, and 2 years for secondary or latent syphilis), and this may help to identify sexual networks with a high risk of future transmission. Empirical treatment for partners may be recommended until negative results of investigations are available. With respect to HSV, patients should be given advice on transmission risks and sharing information with partners. Co-infection with other sexually transmitted pathogens is common and so full sexual health screening including HIV, hepatitis B and $\mathrm{C}$ is advisable.

\section{CONCLUSION}

When evaluating a patient with either new-onset disease, proctitis that has not responded to standard treatments for Crohn's disease or ulcerative colitis, or in the presence of atypical clinical or laboratory features, there are several differential diagnoses outlined by this paper that must be considered. Box 2 describes common or key discriminating features of these conditions with respect to Crohn's disease and ulcerative colitis. Box 3 outlines risk factors for STIs that should be considered by clinicians, and box 4 advises on taking a sexual history in the gastroenterology clinic. Sexual health experts should be consulted in cases of confirmed or suspected STI, when further screening for co-infection including HIV, hepatitis B and C should then be undertaken, along with tests of cure and partner identification as appropriate.
Box 2 Common or key discriminating features from Crohn's disease or ulcerative colitis

Gonorrhoea

- Purulent urethral discharge

- Pharyngeal infection

- Gram-negative diploccoci on Gram stain of anogenital swabs

- Chlamydia co-infection common

Lymphogranuloma venereum

- Mucopurulent anal, urethral or vaginal discharge

- Painful inguinal or femoral lymphadenopathy possibly with associated abscess formation

- Low-grade fever and pyrexia

- C trachomatis biovar L1, L2 or L3 identification on nucleic acid amplification test and genotyping

Chlamydia

- Proctitis symptoms usually mild (often asymptomatic)

- Neutrophils may be seen on microscopy of anal discharge

- Gonorrhoea co-infection common

Herpes simplex virus

- Malaise, headaches and myalgia

- Perianal vesicles or ulcers

- Painful genital ulceration

- HSV type 1 or 2 identification on PCR

- Syphilis

- Painless rectal or orogenital chancre (primary disease)

- Snail tract oral ulceration

- Condylomata lata

- Associated neurological, cognitive and vascular co-morbidities (secondary and tertiary disease)

- Gastrointestinal or glutaeal gummatous ulceration or nodules

Behçet's syndrome

- Punched-out oral ulceration

- Proctocolonic ulcers may not have surrounding proctocolitis.

- Lower limb thrombophlebitis

- Genital ulceration

- Eye and skin lesions common

- Positive pathergy test

Solitary rectal ulcer syndrome

- Rectal prolapse

- Smooth muscle extension from muscularis into lamina propria and relative absence of inflammatory infiltrate on histology

\section{BEST OF FIVE QUESTIONS}

\section{Question 1}

A 23-year-old man presented with an 8-week history of bloody diarrhoea, severe anorectal pain and lowgrade pyrexia. On further questioning he also complained of malaise, myalgia and arthralgia. He admitted to unprotected receptive anal sexual intercourse with two male partners in the preceding 6 months. Perianal and rectal examination revealed two fluctuant, tender areas of induration adjacent to the anal verge suggestive of abscesses. An urgent flexible sigmoidoscopy revealed inflamed, friable rectal mucosa

Box 3 Risk factors associated with sexually transmitted infections ${ }^{11}$

Younger age (less than 25 years at highest risk)

- People from or who have visited countries with higher rates of STI

- Men who have sex with men

- Frequent partner change or multiple concurrent partners

- Early onset sexual activity

- Previous STI or previous contact with STI

- Alcohol or substance misuse 
Box 4 Components of and tips for taking a sexual history in the gastrointestinal clinic ${ }^{12}$

Components

- Symptoms: Duration, association with genital or bladder symptoms, presence and colour of discharge

- Partners: Last sexual intercourse, partner gender, establish number of partners within preceding 3-6 months, partner symptoms, type of sexual intercourse (oral, vaginal or anal)

- Contraception: Specifically the use of condoms

- History of STI: Previous diagnoses and treatment of STI or partner diagnoses/ treatment

- Foreign travel: Travel to areas of high incidence

- Social history: Including alcohol and drug misuse

- For women: Last menstrual period

Tips

- Speaking to the patient alone may allow them to speak more openly about their symptoms and allow you to ask more detailed questions, as can utilising a sexual history proforma, used in many GUM clinics

- Seek permission and explain why you are asking personal questions

- Only ask what you need to know, avoid needlessly intrusive questions

with several large ulcers and spontaneous bleeding. Rectal swabs sent for NAAT and genotyping revealed the presence of $C$ trachomatis genotype L1.

Which of the following is the most appropriate treatment regimen:

A-Doxycycline $100 \mathrm{mg}$ twice a day for 7 days

B-Erythromycin $250 \mathrm{mg}$ four times a day for 21 days

C-Olfloxacin $400 \mathrm{mg}$ a day for 7 days

D-Erythromycin $500 \mathrm{mg}$ twice a day for 7 days

E-Doxycycline $100 \mathrm{mg}$ twice a day for 21 days

\section{Question 2}

A 32-year-old man presents with a 6-week history of diarrhoea, anorectal pain and maculopapular rash affecting the trunk, hands and feet. Two years previously he had been investigated for possible Crohn's disease after presenting with several perianal and anal verge ulcers. These had resolved spontaneously and no formal diagnosis had been made. On further questioning he admitted to a 4-week history of perianal swelling and several oral ulcers with a snail track distribution on inspection. Perianal inspection is demonstrated in figure 3.

What is the most likely causative organism:

$$
\text { A-HSV }
$$

B-T pallidum

C-N gonorrhoea

$\mathrm{D}-\mathrm{C}$ trachomatis

E-Human papilloma virus

\section{Question 3}

A 40-year-old male patient of Turkish origin presented to ophthalmology as an emergency with acute painless reduced visual acuity in both eyes. Further questioning revealed a several year history of intermittent diarrhoea, recurrent oral and penile ulceration, with orogenital scarring evident on examination. $\mathrm{He}$ also complained of painful red nodules on the extensor aspects of both forearms. One year previously he had been diagnosed with anterior uveitis requiring prolonged steroid use. Slit lamp retinal examination revealed multiple intraretinal haemorrhages, soft exudates and macular oedema in both eyes.

What is the most likely diagnosis:

A-Crohn's disease

B-Sarcoidosis

C-Behçet's syndrome

D-Tuberculosis

E-Multiple sclerosis 


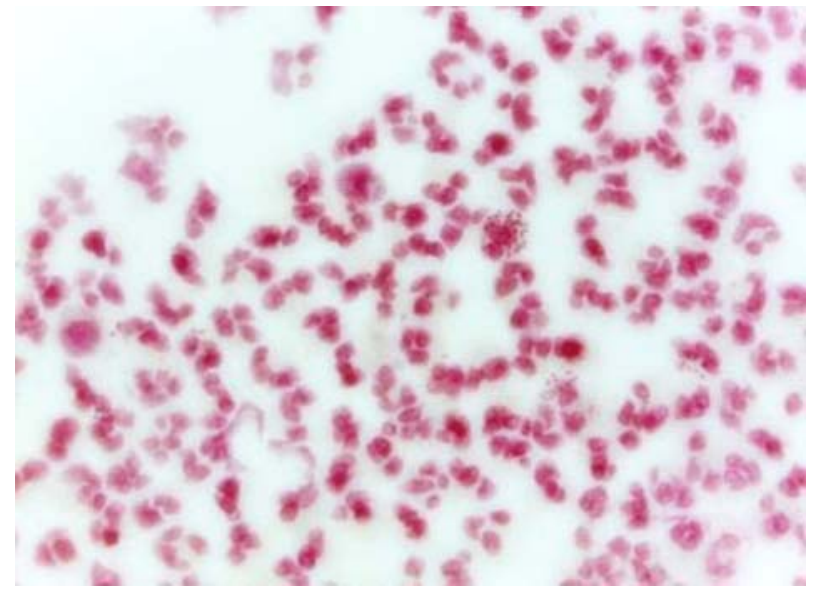

Figure 4 Gram stain of swab exudate (question 4).

\section{Question 4}

A 32-year-old woman presented to the endoscopy department for flexible sigmoidoscopy with a 1-month history of diarrhoea and abdominal pain. Two months previously she had been treated in primary care for chlamydia infection following a positive urine NAAT. Symptoms of dysuria and urethral discharge had continued despite 7 days doxycycline $100 \mathrm{mg}$ twice a day. Endoscopy revealed mildly erythematous mucosa and loss of normal vascular pattern in the rectum associated with a purulent discharge. Anal and urethral swabs were obtained and the resultant Gram stain is shown in figure 4.

What is the most likely causative organism:

\author{
A-C trachomatis \\ B-HSV \\ C-Human papilloma virus \\ D-T pallidum \\ E-N gonorrhoea
}

\section{ANSWERS}

\section{Answer 1: E}

The symptoms described and positive testing for $C$ trachomatis serovar L1 are diagnostic of LGV. While a 7 -day course of doxycycline $100 \mathrm{mg}$ twice a day, olfloxacin $400 \mathrm{mg}$ a day or erythromycin $500 \mathrm{mg}$ twice a day would be appropriate to treat uncomplicated serovar D-K C trachomatis, LGV caused by invasive serovar $\mathrm{L} 1,2$, or 3 requires 21 days of therapy, and while erythromycin would be appropriate, a higher dose of $500 \mathrm{mg}$ four times a day would be required. The correct answer is therefore doxycyline $100 \mathrm{mg}$ twice a day for 21 days.

\section{Answer 2: B}

The depicted flat-topped, papillomatous perianal lesions are typical of condylomata lata, a feature of secondary syphilis caused by $T$ pallidum. The described oral ulceration and macular rash with a palmoplantar distribution are also typical of secondary syphilis. The previous perianal ulcers are likely to have represented multiple primary syphilitic chancres, as although these are classically singular, multiple lesions may be seen.

\section{Answer 3: C}

The ocular findings are consistent with retinal vasculitis that can be confirmed by fluorescein angiography. The painful nodules on his forearms are suggestive of erythema nodosum, and these diagnoses associated with previous anterior uveitis, recurrent orogenital ulceration and gastrointestinal symptoms are consistent with Behçet's syndrome. The highest prevalence of Behçet's syndrome is in Turkey. Tuberculosis, sarcoidosis and Crohn's disease are among the differential diagnoses; however, recurrent genital ulceration would be uncommon and is more typically a finding of Behçet's syndrome. Orogenital ulceration is not a feature of multiple sclerosis.

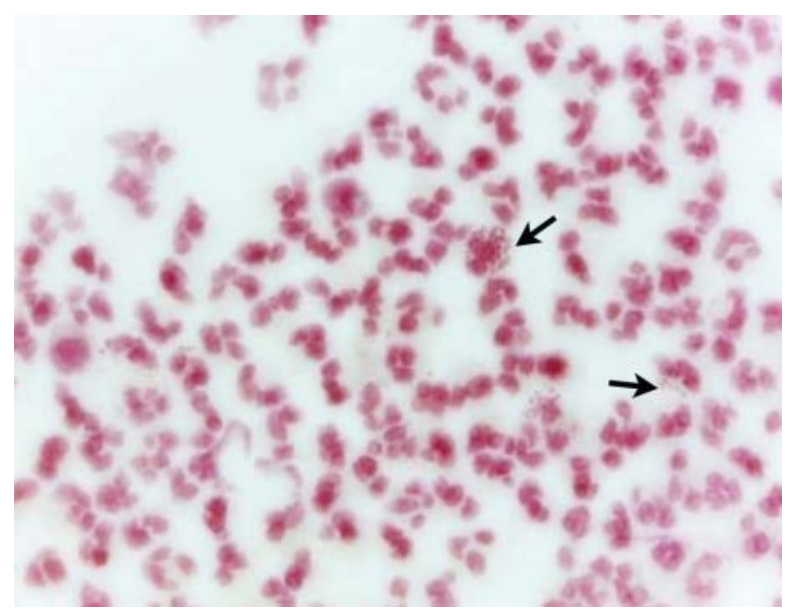

Figure 5 Gram stain of swab exudate (answer 4). Arrows point towards neutrophils containing intracellular Gram-negative diplococci. 


\section{Answer 4: E}

The Gram stain reveals numerous neutrophils, many with intracellular Gram-negative diplococci (arrows in figure 5) consistent with $N$ gonorrhoea infection. Co-infection with gonorrhoea is commonly associated with chlamydia, and gonorrhoea would not have responded to the previous course of doxycycline, hence the patient's ongoing symptoms. Rectal infection with gonorrhoea can occur due to the transmucosal spread of infected urethral discharge. Appropriate therapy for gonorrhoea proctitis includes ceftriaxone $500 \mathrm{mg}$ intramuscularly once, plus oral azithromycin $1 \mathrm{~g}$ once.

Contributors CAL devised the concept for the manuscript, which was subsequently developed by CAL and EIML. Initial drafting was undertaken by CAL, all authors contributed to subsequent drafts. KNS supplied the colour images used in this paper.

Patient consent Obtained.

Funding None.

Competing interests None.

Provenance and peer review Not commissioned; internally peer reviewed.

\section{REFERENCES}

1 Health Protection Agency. New data show sexually transmitted infection diagnoses on the rise in England. HPA Press release, 31st May 2012. http://www.hpa.org.uk/NewsCentre/ NationalPressReleases/2012PressReleases/ 120531newrisingSTInumbersreleased/ (accessed 15 Oct 2012).
2 Bignell C, Fitzgerald M. UK national guideline for the management of gonorrhoea in adults, 2011. Int J STD AIDS 2011;22:541-7.

3 McMillan A, van Voorst Vader PC, de Vries HJ. The 2007 European Guideline (International Union against Sexually Transmitted Infections/World Health Organization) on the management of proctitis, proctocolitis and enteritis caused by sexually transmissible pathogens. Int J STD AIDS 2007; 18:514-20.

4 de Vries HJ, Morre SA, White JA, et al. European guideline for the management of lymphogranuloma venereum, 2010. Int J STD AIDS 2010;21:533-6.

5 Patel R, Alderson S, Geretti A, et al. European guideline for the management of genital herpes, 2010. Int J STD AIDS 2011;22:1-10.

6 French P, Gomberg M, Janier M, et al. IUSTI: 2008 European guidelines on the management of syphilis. Int J STD AIDS 2009;20:300-9.

7 Shelton AA. Sexually transmitted parasitic diseases. Clin Colon Rectal Surg 2004;17:231-4.

8 Yazici H, Fresko I, Yurdakul S. Behcet's syndrome: disease manifestations, management, and advances in treatment. Nat Clin Pract Rheumatol 2007;3:148-55.

9 International Study Group for Behcet's Disease. Criteria for diagnosis of Behcet's disease. Lancet 1990;335: 1078-80.

10 Vaizey CJ, van den Bogaerde JB, Emmanuel AV, et al. Solitary rectal ulcer syndrome. Br J Surg 1998;85:1617-23.

11 Clutterbuck DJ, Flowers P, Barber T, et al. UK national guideline on safer sex advice. Int J STD AIDS 2012;23:381-8.

12 French P. BASHH 2006 National Guidelines-consultations requiring sexual history-taking. Int J STD AIDS 2007;18:17-22. 